\title{
Medical Student Schwartz Rounds: A Powerful Medium for Medical Student Reflective Practice
}

This article was published in the following Dove Press journal:

Advances in Medical Education and Practice

\section{David Gleeson (D) James Arwyn-Jones (D) Mariam Awan (D) Isabel White (iD Omid Halse (D)}

Medical Education, Charing Cross Hospital, Imperial College London, London, UK
Correspondence: David Gleeson Charing Cross Hospital, Imperial College London, Fulham Palace Rd,

Hammersmith, London W6 8RF, UK Tel +44020 33II I234

Email David.gleeson@doctors.org.uk
Purpose: Reflection is a core component of good medical practice and of growing importance given increasing rates of burnout in the field of medicine. Schwartz Rounds (SRs) are a group-based model of reflective practice. This study explored the utility of student-specific Schwartz Rounds as a medium for reflective practice amongst medical students entering their first year of clinical attachments, and how effective these are compared to standard organisation-wide Schwartz Rounds.

Methods: Three Medical Student Schwartz Rounds (MSSRs) were piloted over the course of a year at a major teaching hospital, focussed on the theme: "Is this what I signed up for?" Participants were asked to complete a questionnaire following the session, and this data was then analysed.

Results: Feedback was obtained from 93\% (42/45) students who attended. Ninety per cent of students rated the sessions as "excellent or exceptional", 93\% felt the MSSRs added to their "insight and self-awareness", and 90\% plan to attend SRs again in the future. A thematic analysis of the qualitative feedback highlighted three broad themes from the pilot; group reflection is more profound than individual reflection, sharing experiences facilitated a greater sense of belonging, and group reflective practice can be intimidating. There was a statistically significant difference between students' ratings of MSSRs, rated $8.67 / 10$, versus written reflection, rated 4.64/10 $(\mathrm{p}<0.01)$.

Conclusion: Students were overwhelmingly positive about their experiences in this pilot, with response ratings commensurate with those from organisation-wide Schwartz Rounds. Therefore, MSSRs should be considered as a valid alternative form of reflective practice.

Keywords: medical education, reflective practice, group-based reflection, Schwartz Rounds

\section{Introduction}

Reflection forms one of the cornerstones of the GMC Good Medical Practice and, as such, is a necessary requirement in appraisal and revalidation processes. ${ }^{1}$ Students are encouraged to engage in reflection early on in order to embed this behaviour into their personal practice. However, there is general dissatisfaction with written reflection, with some seeing it as a waste of time, and a further unnecessary requirement of training. ${ }^{2}$ Furthermore, the recent high profile case of Dr BawaGarba, whose written reflections were subpoenaed, has led to further uneasiness with the reflective process. ${ }^{3}$

Morale within the NHS is at crisis point, with a quarter of doctors reporting feeling burnt-out, and one out of eleven posts currently lying vacant. ${ }^{4}$ The problem now extends into the medical student population, with burnout rates amongst this cohort rising, and empathy rates falling over the course of training. ${ }^{5}$ Much focus 
has turned to different types of resilience training in order to boost workforce morale, and one such strategy is the implementation of Schwartz Rounds (SRs).

SRs were developed in the US over 25 years ago and were inspired by a patient, Kenneth Schwartz, who died at the age of 39 with lung cancer. SRs were initially developed to promote compassion in healthcare and to allow multi-disciplinary professionals to come together and reflect on the emotional aspects of working in healthcare. Each Round focuses on a particular theme or case, with panel members each sharing a relevant story and discussing its impact on them, before opening up to an audiencewide, confidential discussion. Crucially, these Rounds are not a problem-solving exercise, but rather a space for reflection and processing of emotions. The most thorough evaluation of Schwartz rounds was recently undertaken as a multisite, prospective NIHR study within in the UK. ${ }^{6}$ The initial report includes evidence that the psychological wellbeing of staff who attended Rounds regularly significantly improved. The proportion of staff with significant psychological distress was reduced from $25 \%$ to $12 \%$ amongst regular attendees, compared to no change in the scores of staff who did not attend (37\% to $34 \%$ ). A recent GMC report on medical staff wellbeing lists Schwartz Rounds as an effective intervention to foster a compassionate and supportive culture. ${ }^{7}$ Likewise, the recently published NHS Workforce Health and Wellbeing Framework lists Schwartz Rounds as a prime opportunity to engage in reflective practice as part of a wider mental health initiative. ${ }^{8}$

Given the current dissatisfaction with written reflective practice, coupled with the positive benefits SRs offer to attendees, we sought to explore whether there is a role for an SR tailored specifically to medical students. A previous study looked into SRs for medical students entering their final years of medical school and explored their feelings of preparedness for practice. ${ }^{9}$ This pilot received very positive feedback and highlighted the need for further consideration regarding the timing and format of these Rounds. We decided to explore the perspectives of students entering their first year of clinical attachments, a key transitional phase in their medical education. Students are exposed to a wealth of new experiences during this period, and we identified this as an opportunity to explore the impact reflective practice could have on the students, whilst allowing them to benefit from the support derived from the SR experience.

\section{Materials and Methods}

\section{Participants and Setting}

Year 3 medical students on rotation through a major UK teaching hospital were invited to attend an hour-long MSSR in the final two weeks of their clinical attachment. Attendance was encouraged but was not mandatory. This model was repeated during the following two attachments in the academic year. The theme for each of these Rounds was: "Is this what I signed up for?" Volunteer panel members, who received facilitator-led preparation in advance, spoke at the beginning of the session, and then there was an open floor-wide discussion. The content of these discussions is confidential.

\section{Data Collection and Measures}

Feedback was obtained using an adapted evaluation form from the Point of Care Foundation. A further short questionnaire was also used asking students to rate the utility of written reflection practice and the MSSR experience, and comment on their preference. All feedback was anonymous. Completion of the feedback forms was voluntary.

\section{Analysis}

Quantitative data were analysed using Microsoft Excel. The results between students' perceptions of written reflective practice and MSSRs were analysed using the paired $t$-test. Qualitative data from white-space comments were entered into Excel, coded, and analysed to identify the key themes, in line with the process outlined by Braun and Clarke. ${ }^{10}$ This was carried out independently by two members of our team, with the results cross-correlated afterwards.

\section{Results \\ Attendance}

Attendance at the MSSRs was voluntary. Overall, 45/84 students $(53 \%)$ attended over the course of the year. One of the Rounds unfortunately clashed with a number of other teaching opportunities, and so was poorly attended, with only $10 / 28$ students $(36 \%)$. Feedback was received from $42 / 45$ attendees (93\%).

\section{Post-Round Quantitative Evaluation}

Students were asked to fill out an adapted Point of Care Foundation evaluation form after attending the Round. Students were asked to give responses ranging from "completely disagree" through to "completely agree" to a range of statements. The percentage of students responding with "completely agree" and "agree" to each statement is listed 
below (Table 1). Data from the corresponding time period of September 2018 to August 2019 of trust-wide Schwartz Rounds across Imperial College NHS Trust (ICNHT) are given as a comparison. A full breakdown of the responses for each question listed in Table 1 is shown in Figure 1. Of note, $93 \%$ of students felt the discussions were useful, and added to their self-awareness and personal insight, whilst $100 \%$ felt the Rounds added to their understanding of how their colleagues felt about their work. Crucially, 90\% would recommend Schwartz Rounds to their colleagues, and "plan to attend Schwartz Rounds again", and $90 \%$ of students rated the Rounds as "excellent or exceptional".

\section{Post-Round Qualitative Evaluation}

Three broad themes emerged from the white-space comments received from the students. These are displayed in Figure 2 with representative quotes.

Table I Percentage of Respondents Answering "Completely Agree" or "Agree" to the Following Questions

\begin{tabular}{|l|l|l|}
\hline Question & $\begin{array}{l}\text { MSSR } \\
\text { Pilot } \\
\text { Rounds }\end{array}$ & $\begin{array}{l}\text { ICNHT } \\
\text { Rounds }\end{array}$ \\
\hline $\begin{array}{l}\text { The stories presented by the panel were } \\
\text { relevant to my daily work }\end{array}$ & $98 \%$ & $94 \%$ \\
\hline $\begin{array}{l}\text { I gained insights that will help me care for } \\
\text { patients }\end{array}$ & $80 \%$ & $89 \%$ \\
\hline $\begin{array}{l}\text { Today's Round will help me work better } \\
\text { with my colleagues }\end{array}$ & $80 \%$ & $91 \%$ \\
\hline The group discussion was helpful to me & $93 \%$ & $95 \%$ \\
\hline The group discussion was well facilitated & $98 \%$ & $97 \%$ \\
\hline $\begin{array}{l}\text { I have a better understanding of how my } \\
\text { colleagues feel about their work }\end{array}$ & $100 \%$ & $95 \%$ \\
\hline $\begin{array}{l}\text { I have a better understanding of how I feel } \\
\text { about my work }\end{array}$ & $80 \%$ & $84 \%$ \\
\hline \begin{tabular}{l} 
I plan to attend Schwartz Rounds again \\
\hline $\begin{array}{l}\text { I would recommend Schwartz Rounds to } \\
\text { colleagues }\end{array}$
\end{tabular} & $90 \%$ & $96 \%$ \\
\hline $\begin{array}{l}\text { Today's Round has added to my insight } \\
\text { and self-awareness }\end{array}$ & $93 \%$ & $94 \%$ \\
\hline $\begin{array}{l}\text { I feel more connected to my colleagues } \\
\text { due to Today's Round }\end{array}$ & $88 \%$ & $91 \%$ \\
\hline Overall rating as "excellent or & $90 \%$ & $78 \%$ \\
\hline
\end{tabular}

\section{Comparison with Written Reflective Practice}

Both quantitative and qualitative data were obtained from students regarding their perceptions of reflective practice. Students were asked to rate the utility of both written reflective practice and the MSSR experience out of 10 , select their preferred type of reflection, and comment on this preference.

There was a statistically significant difference between the average ratings of the two forms of reflective practice, as shown in Figure 3. Furthermore, 38/42 students (90\%) selected MSSRs as their preferred method of reflective practice, with just one student saying they preferred written reflection, and three students rating them equally (Figure 4).

\section{Discussion}

\section{Impact}

From the feedback received, this early data indicates that students found the MSSRs to be impactful, improving perceptions of self-awareness and insight, whilst helping to foster closer working relationships with colleagues through shared experiences. Ninety per cent of students rated the sessions as "excellent or exceptional", with $90 \%$ planning to re-attend SRs in the future. These results closely correlated to the highly successful organisationwide Schwartz Rounds already in operation, and give strong evidence in support of the role of MSSRs moving forwards. These results also closely tally with the preliminary results from a similar student-focused study being run through the University of Surrey, indicating a growing body of evidence justifying their use. ${ }^{11}$

Students are often invited to attend hospital-wide SRs but these can be a daunting environment for some young students given the range of seniority present. We hope that an early and graduated introduction to the format and nature of the Rounds will translate into greater attendance and participation in future multi-disciplinary Rounds.

From the students' comments, it was apparent that there was no similar structured approach to group reflection readily available. One student described attending the Round as the "best experience in [their] clinical attachment", whilst multiple students described it as a "safe space to reflect". We feel the feedback received is a clear validation of MSSRs as a tool for reflective practice amongst medical students, providing a novel and powerful medium that is currently under-utilised in the delivery of undergraduate teaching. 


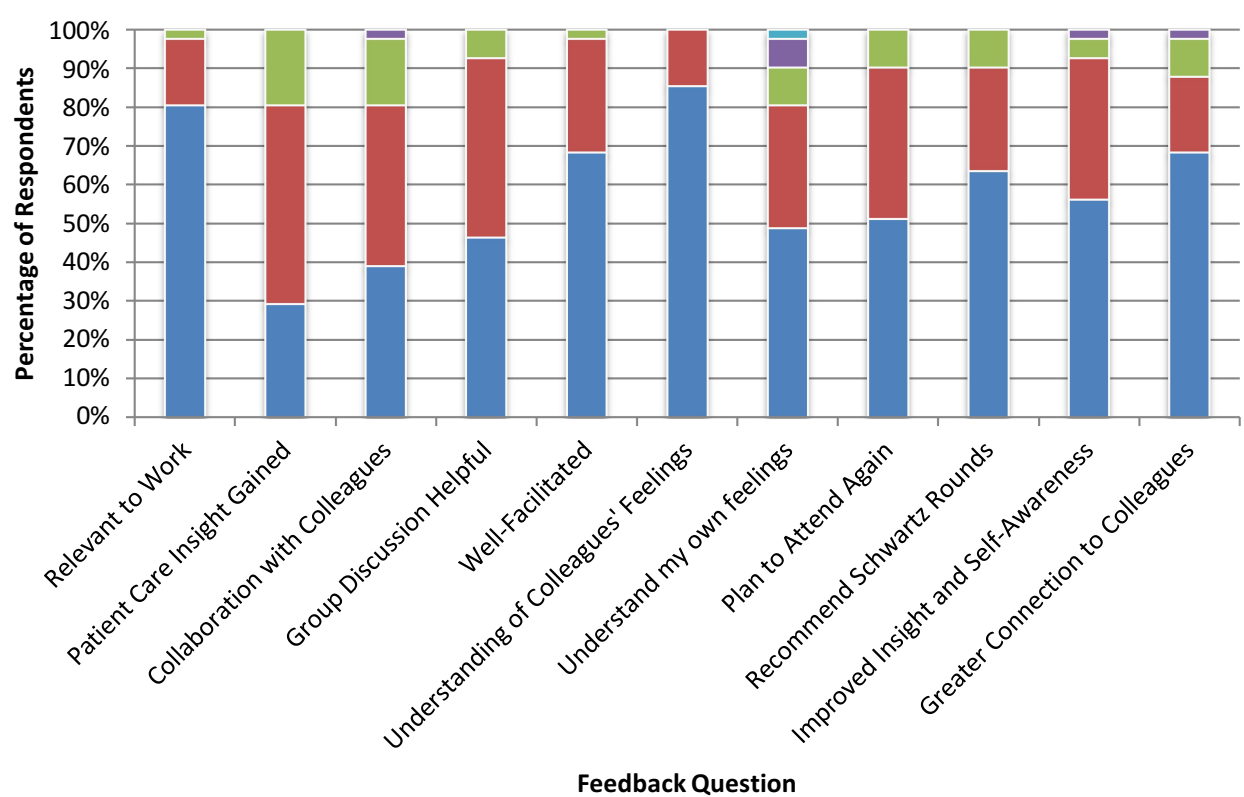

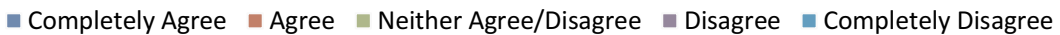

Figure I Breakdown of responses to each question in the feedback form.
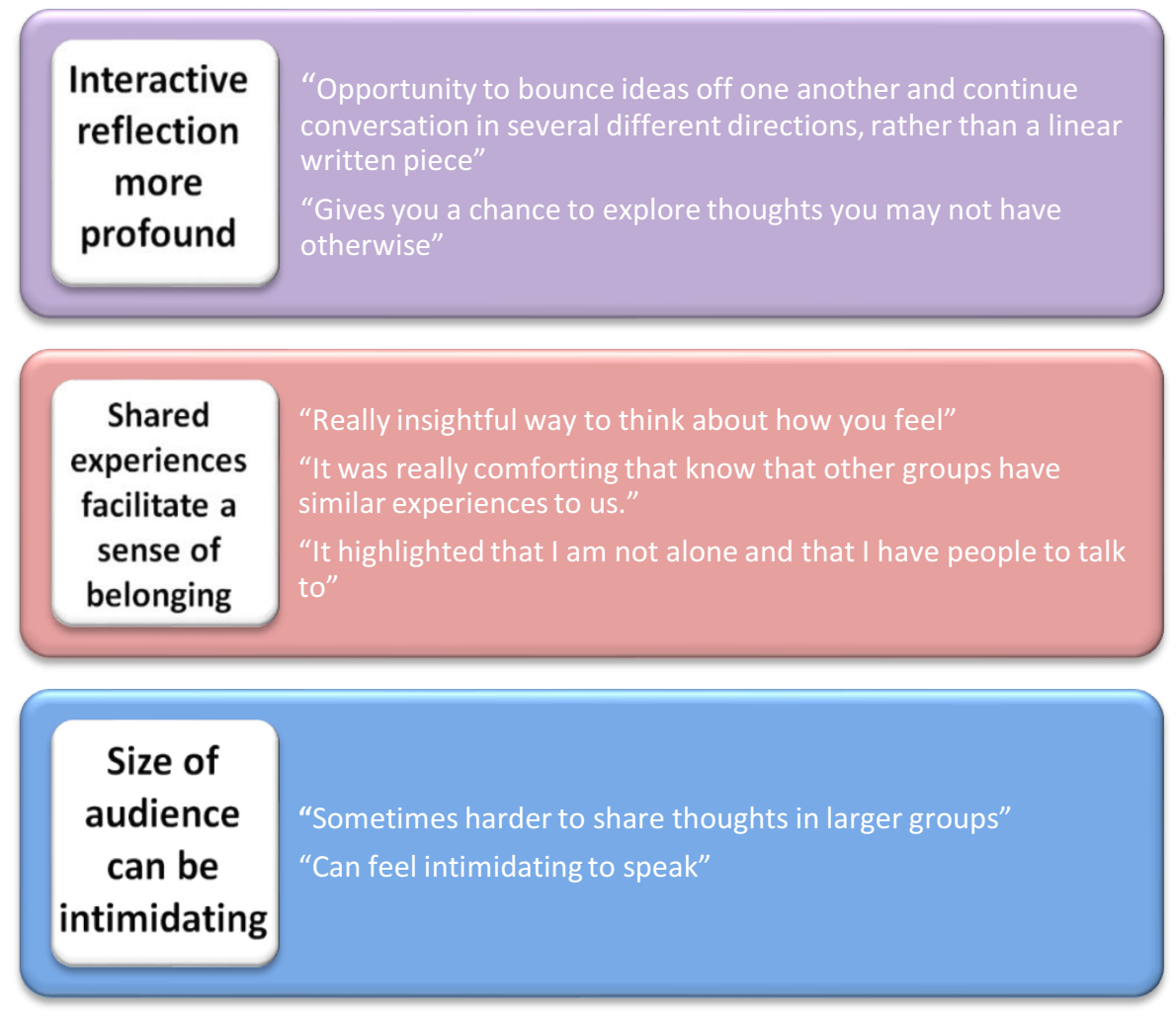

Figure 2 Thematic analysis of the feedback received. 


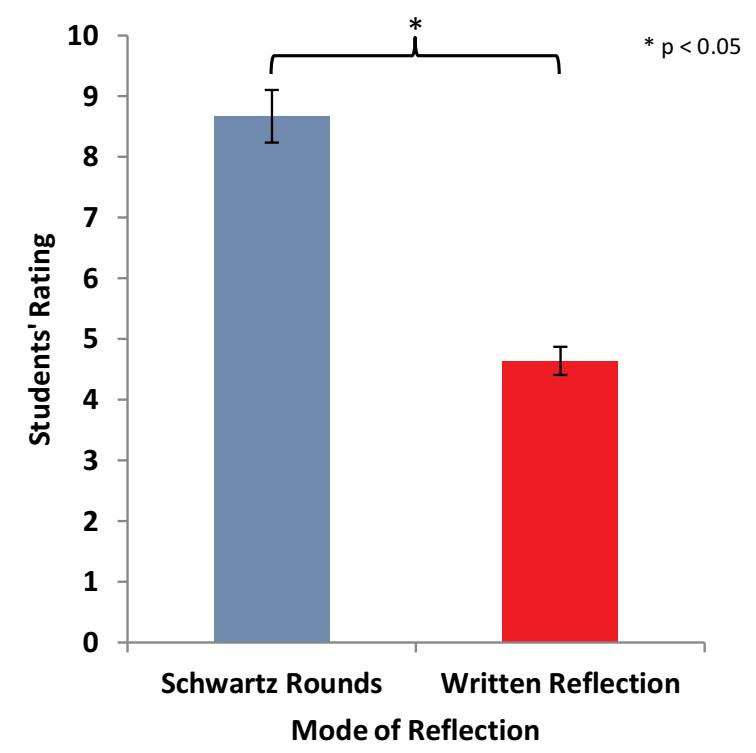

Figure 3 Respondents' perceptions of utility of written reflection versus Schwartz Rounds out of 10.

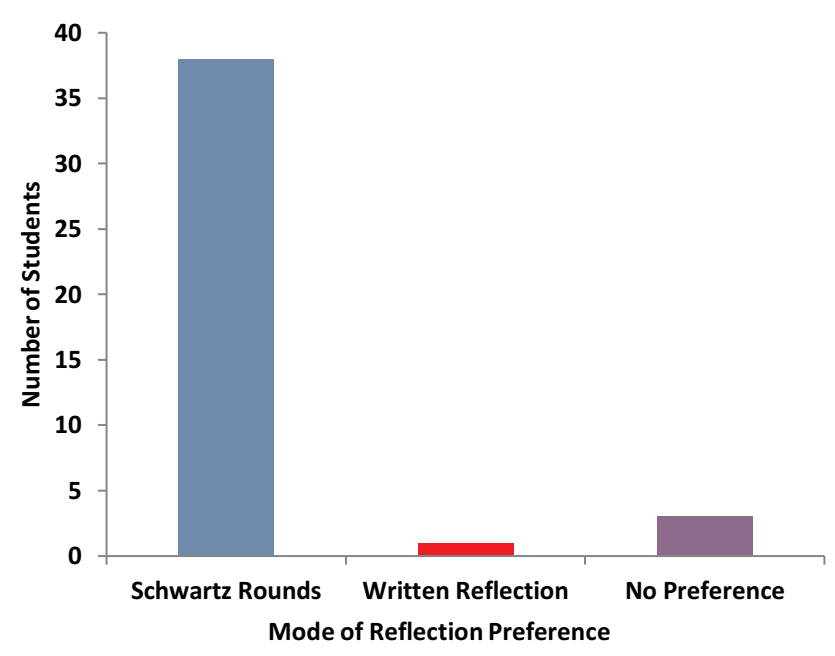

Figure 4 Respondents' preferred mode of reflective practice.

\section{Limitations}

This pilot was not without its difficulties, and the financial and logistical implications of running MSSRs may hinder other sites being able to introduce them. In the UK, SRs are licensed through the Point of Care Foundation, with a setup cost of $£ 5000$, and a $£ 1250$ membership fee thereafter. Furthermore, hour-long panellist preparation and debrief sessions must also be factored in, meaning facilitating the session can be a big undertaking. The availability of local facilitators across the country is highly variable, posing a key barrier to their introduction for some medical schools.
A key issue encountered during the first MSSR was a lack of understanding regarding the function of the Round. SRs are not a forum for raising concerns or problem-solving, and some of the initial discussion veered towards students asking the facilitators how they should have approached certain situations or dealt with certain issues. Moving forwards, the aims and function of the MSSRs were more explicitly stated to the attendees, and alternate avenues for raising concerns were highlighted to students. This meant attendees' expectations of the Round more closely allied with their experiences, and the discussion was more reflective and impactful.

It is also key to note that, whilst the vast majority of students stated a preference for MSSRs over written reflection, three students rated them equally, and one rated written reflection more highly. Furthermore, one of the key recurring themes from the qualitative analysis was that the group setting may be intimidating for some students, preventing them from participating. Reflective practice should be an integral part of a student's development, and should not be limited to single events or activities. We do not envisage MSSRs replacing all other forms of reflective practice, but rather that they are integrated to supplement and enhance the other more traditional methods already in place.

\section{Moving Forward}

This study focussed on the role of Schwartz Rounds as a form of reflective practice for students entering the key transition into clinical attachment at medical school. A clear preference was expressed for Schwartz Rounds over written reflection, and there is scope for more exploration of this finding. Is it specific to the structured, confidential format of the Schwartz Round, which enabled students to feel freer to speak openly about their experiences? Or would students prefer other types of group reflection just as much, and also display a preference for these over written reflection?

Furthermore, it would be interesting to know whether early exposure to SRs during medical school affects students' future attendance rates at SRs. The feedback received during our study indicated a strong willingness to attend future Rounds. A key finding in the NIHR study of SRs found an increase in wellbeing amongst regular attendees of SRs. Does this finding translate to the medical school population, and, if so, could MSSRs be employed to help reduce burnout amongst medical students? Would early exposure to this format of reflective practice 
encourage ongoing attendance beyond medical school, helping to foster a more resilient, empathetic workforce?

\section{Conclusion}

SRs are now well recognised as having a significant positive impact on the wellbeing of those who attend. A previous study looking at fifth- and sixth-year medical students indicated MSSRs might have a valid role as part of the undergraduate curriculum. ${ }^{9}$ Our study gives more weight to this viewpoint, and in particular looks at the benefits MSSRs can give to medical students earlier on in their training. Students displayed a clear preference for group reflection as opposed to individual written reflection and felt it was more impactful. Given the financial and logistical implications of running Rounds and the potentially intimidating nature of speaking in front of a group, we do not feel that MSSRs should ever replace other forms of reflection, but that they are an extremely useful and currently under-utilised adjunct.

\section{Ethics}

This project proposal was discussed with the Medical Education Ethics Committee, Imperial College London, on the $11 / 3 / 2019$, and it was agreed ethical approval was not required.

\section{Acknowledgments}

We wish to acknowledge the input of the Schwartz Rounds team at Imperial College Healthcare NHS Trust, including Dr Paquita De Zulueta, Dr Karen Frame, Mr Julian Henty, Dr Alex King, Dr Alex Orchard, Dr Joanne Thompson and Dr Matthew Williams, for their help in facilitating the Rounds, and their advice throughout the research process.

\section{Funding}

No funding to declare.

\section{Disclosure}

The authors report no conflicts of interest for this work.

\section{References}

1. GMC | Good Medical Practice. GMC, United Kingdom. 2019. Available from: http://www.gmc-uk.org/guidance/good_medical_prac tice.asp. Accessed June 28, 2020.

2. Mann KV, Gordon J, MacLeod A. Reflection and reflective practice in health professions education: a systematic review. Adv Health Sci Educ Theory Pract. 2007;14:595-621. doi:10.1007/s10459-0079090-2

3. Hodson N. Reflective practice and gross negligence manslaughter. $\mathrm{Br}$ J Gen Pract. 2019;69(680):13. doi:10.3399/bjgp19X701561

4. GMC. The State of Medical Education and Practice in the UK. United Kingdom: GMC; 2018. Available from: https://www.gmcuk.org/-/media/documents/the-state-of-medical-education-andpractice-in-the-uk—workforce-report_pdf-80449007.pdf. Accessed June 28, 2020.

5. Ishak W, Nikravesh R, Lederer S, Perry R, Ogunyemi D, Bernstein C. Burnout in medical students: a systematic review. Clin Teach. 2013;10(4):242-245. doi:10.1111/tct.12014

6. Maben J. A Realist Informed Mixed Methods Evaluation of Schwartz Center Rounds in England. NIHR; 2017.

7. GMC. Caring for Doctors Caring for Patients. United Kingdom: GMC; 2019. Available from: https://www.gmc-uk.org/-/media/docu ments/caring-for-doctors-caring-for-patients_pdf-80706341.pdf. Accessed June 28, 2020.

8. NHS Improvement. Workforce Health and Wellbeing Framework. United Kingdom: NHS Improvement; 2019. Available from: https:// improvement.nhs.uk/resources/workforce-health-and-wellbeingframework/. Accessed June 28, 2020.

9. Gishen F, Whitman WS, Gill D, Barker R, Walker S. Schwartz centre rounds: a new initiative in the undergraduate curriculum-what do medical students think? BMC Med Educ. 2016;16(1). doi:10.1186/ s12909-016-0762-6

10. Braun V, Clarke V. Using thematic analysis in psychology. Qual Res Psychol. 2008;3(2):77-101. doi:10.1191/1478088706qp063oa

11. Jakimowicz S, Maben J. "I can't stop thinking about it": Schwartz rounds ${ }^{\circledR}$ an intervention to support students and higher education staff with emotional, social and ethical experiences at work. J Clin Nurs. 2020. doi:10.1111/jocn.15354
Advances in Medical Education and Practice

\section{Publish your work in this journal}

Advances in Medical Education and Practice is an international, peerreviewed, open access journal that aims to present and publish research on Medical Education covering medical, dental, nursing and allied health care professional education. The journal covers undergraduate education, postgraduate training and continuing medical education including emerging trends and innovative models linking education, research, and health care services. The manuscript management system is completely online and includes a very quick and fair peer-review system. Visit http://www.dovepress.com/testimonials.php to read real quotes from published authors. 